\title{
MicroRNA-125b inhibits cell proliferation and induces cell apoptosis in esophageal squamous cell carcinoma by targeting $\mathrm{BMF}$
}

\author{
YAN-XIN FAN, XIU-HUA BIAN, PU-DONG QIAN, ZHEN-ZHANG CHEN, \\ JING WEN, YAN-HONG LUO, PENG-WEI YAN and QIAN ZHANG
}

Department of Radiotherapy, Jiangsu Cancer Hospital, Jiangsu Institute of Cancer Research, The Affiliated Cancer Hospital of Nanjing Medical University, Nanjing, Jiangsu 210000, P.R. China

Received October 10, 2017; Accepted April 17, 2018

DOI: 10.3892/or.2018.6413

\begin{abstract}
MicroRNAs (miRNAs) have been reported to regulate the expression of genes by suppressing translation or facilitating mRNA decay. Their expression regulates a wide variety of cellular processes, including the development and progression of cancer. Esophageal squamous cell carcinoma (ESCC) is a malignant cancer with high morbidity and recurrence in Asia. In the present study, the biological function of miR-125b and its underlying mechanism in ESCC were explored. The results revealed that miR-125b expression was significantly decreased in ESCC tissues and cell lines. A decrease in miR-125b was markedly related to lymphatic metastasis in patients. Functional analysis revealed that the overexpression of miR-125b using miR-125b mimics significantly inhibited cell growth and induced cell apoptosis, and increased the G1 phase of the cell cycle in EC109 and EC9706 cells. Notably, the miR-125b inhibitors revealed the opposite effect. Additionally, overexpression of miR-125b significantly inhibited tumor growth in vivo. Furthermore, BCL-2-modifying factor (BMF) was considered to be a potential candidate target of miR-125b based on miRNA target databases. miR-125b negatively regulated BMF expression by directly binding to its 3 -untranslated region. BMF was a functional target of miR-125b in the regulation of cell proliferation, cell apoptosis and the cell cycle in EC109 and EC9706 cells. In clinical ESCC specimens, BMF expression was upregulated, and negatively correlated with that of miR125b. In conclusion, miR-125b had an antitumor role in ESCC
\end{abstract}

Correspondence to: Professor Qian Zhang, Department of Radiotherapy, Jiangsu Cancer Hospital, Jiangsu Institute of Cancer Research, The Affiliated Cancer Hospital of Nanjing Medical University, 42 Baiziting Road, Xuanwu, Nanjing, Jiangsu 210000, P.R. China

E-mail: zhangqian0093@163.com

Key words: esophageal squamous cell carcinoma, miR-125b, BMF, proliferation, apoptosis, negative correlation cells mediated by targeting BMF, which can be potentially useful for tumorigenesis in ESCC.

\section{Introduction}

Esophageal carcinoma (EC) is a common gastrointestinal tumor with leading cancer-related mortality $(1,2)$. Notably, as a main subtype of esophageal carcinoma, the incidence of ESCC is particularly high in China, with $1.5 \times 10^{5}$ cases of mortality annually reported $(3,4)$. The carcinogenesis of ESCC is considered to be a multi-factor and multi-step process (5). Although medical and surgical techniques have improved, the prognosis for ESCC is unsatisfactory, and the 5-year survival rate of ESCC patients is in the range of $26.2-49.4 \%(6,7)$. Therefore, it is important to understand the underlying molecular mechanisms and find novel molecular markers associated with ESCC.

MicroRNAs (miRNAs) are non-coding RNAs, which downregulate the gene expression of their targets by binding to their 3'-untranslated regions (3'-UTRs) $(8,9)$. It has been revealed that miRNAs play important roles in multiple cellular physiological processes, including cell proliferation, apoptosis and differentiation $(10,11)$. Aberrantly expressed miRNAs are known to be closely associated with tumor development and progression via the regulation of cell growth, drug resistance and metastasis (12). miR-183, as a tumor-promoter, was reported to enhance human ESCC cell proliferation and invasion (13), while miR-486-5p was found to exert antitumor effects by regulating the between cell proliferation and apoptosis in cancers, such as NSCLC (14), breast cancer (15) and hepatocellular carcinoma (HCC) (16). Collectively, miRNAs have been recognized as diagnostic biomarkers for cancers.

miR-125b, a tumor-suppressor miRNA, suppressed cell proliferation and differentiation by affecting Hedgehog signaling in cerebellar neuronal progenitor and tumor cells (17). Overexpression of miR-125b also suppressed the level of Bak1 and induced prostate cancer cell proliferation (18). Additionally, miR-125b exhibited its antitumor effects in colorectal (19) and breast cancer (20). Recent studies have revealed that miR-125b expression was decreased in ESCC 
tissues (21). However, the correlation between miR-125b and pathogenesis of ESCC still remains unknown. Herein, we analyzed the underlying mechanisms of action of miR-125b, resulting in tumor suppression in ESCC.

\section{Materials and methods}

ESCC tissue collection. Human esophageal tumor tissues and control normal tissues were simultaneously isolated from 66 patients between January 2015 and December 2016. All patients underwent esophagectomy without chemotherapy or radiotherapy at the Department of Gastroenterology at Jiangsu Cancer Hospital. All 66 patients provided written informed consent, and all experimental protocols were approved by the Ethics Committee of Jiangsu Cancer Hospital. Fresh tissues were stored at $-80^{\circ} \mathrm{C}$ until future use.

Cell culture and transfection. In the present study, the human ESCC cell lines (EC109 and EC9706) and human esophageal epithelial cells (HET-1A) were obtained from Riken BioResource Center (Tsukuba, Japan). Cell lines were cultured in Roswell Park Memorial Institute-1640 medium (RPMI-1640; Invitrogen; Thermo Fisher Scientific, Inc., Waltham, MA, USA) supplemented with $10 \%$ fetal bovine serum (FBS; Invitrogen; Thermo Fisher Scientific, Inc.), $100 \mathrm{U} / \mathrm{ml}$ streptomycin and $100 \mathrm{U} / \mathrm{ml}$ penicillin (Invitrogen; Thermo Fisher Scientific, Inc.).

miR-125b negative control (NC), mimics, inhibitors and BCL-2-modifying factor (BMF) small interfering RNAs (siRNAs) were purchased from GenePharma Company (Shanghai, China). The aforementioned plasmids were transfected into ESCC cell lines (EC109 and EC9706) and HET-1A using Lipofectamine 2000 (Thermo Fisher Scientific, Inc.)

RNA isolation and quantitative real-time PCR (qRT-PCR). Total RNA was extracted from tissue or cells using TRIzol reagent (Invitrogen; Thermo Fisher Scientific, Inc.) according to the manufacturer's instructions. RNA was reversed-transcribed into cDNA using PrimeScript Reverse Transcription kit (Takara Bio, Inc., Otsu, Japan). The qRT-PCR reactions were performed using SYBR Premix Ex Taq (Takara Bio, Inc.) on a 7500 ABI system (Applied Biosystems; Thermo Fisher Scientific, Inc). U6-siRNA was used for normalization. Specific PCR primers were synthesized at Invitrogen; Thermo Fisher Scientific, Inc. The primer sequences used were as follows: miR-125b, 5'-TCCCTGAGACCTAACTTGTG-3' (forward); U6, 5'-ACGCAAATTCGTGAAGCGTT-3' (forward), a universal primer (reverse); BMF, 5'-CCCAT AAGCCAGGAAGACAA-3' (forward), and 5'-CTGAAGCT TTCTGGCGATTCT-3' (reverse).

Cell proliferation assay. The cell proliferation rate was evaluated using Cell Counting Kit-8 (CCK-8; Dojindo Molecular Technologies, Inc., Kumamoto, Japan), following the manufacturer's instructions. Twenty-four hours after transfection, the cells were seeded into 96-well plates at a density of $\sim 2 \times 10^{3}$ cells/well. The absorbance of each sample was assessed using a microplate reader (Bio-Rad Laboratories, Inc., Hercules, CA, USA) at three different time-points. Each experiment was conducted in triplicate.
Table I. The association between the expression level of miR-125b with the clinical characteristics of ESCC patients.

\begin{tabular}{|c|c|c|c|}
\hline $\begin{array}{l}\text { Clinical } \\
\text { characteristics }\end{array}$ & $\begin{array}{l}\text { No. of } \\
\text { patients }\end{array}$ & $\begin{array}{l}\text { Relative } \\
\text { expression }\end{array}$ & P-value \\
\hline Age (years) & & & 0.681 \\
\hline$\leq 60$ & 35 & 0.364 & \\
\hline$>60$ & 31 & 0.523 & \\
\hline Sex & & & 0.753 \\
\hline Male & 45 & 0.462 & \\
\hline Female & 21 & 0.534 & \\
\hline Smoking & & & 0.712 \\
\hline Yes & 15 & 0.496 & \\
\hline No & 51 & 0.552 & \\
\hline Drinking & & & 0.806 \\
\hline Yes & 18 & 0.409 & \\
\hline No & 48 & 0.598 & \\
\hline pT stage & & & 0.668 \\
\hline $\mathrm{T} 1+\mathrm{T} 2$ & 30 & 0.460 & \\
\hline $\mathrm{T} 3+\mathrm{T} 4$ & 36 & 0.517 & \\
\hline Lymphatic metastasis & & & 0.035 \\
\hline Positive & 23 & 0.226 & \\
\hline Negative & 43 & 0.654 & \\
\hline pTNM stage & & & 0.951 \\
\hline$\leq \mathrm{II}$ & 26 & 0.554 & \\
\hline$>$ II & 40 & 0.512 & \\
\hline
\end{tabular}

Cell apoptosis. A flow cytometer (BD FACSCalibur; BD Biosciences) was used for quantifying the cell apoptosis rates. After transfection for $48 \mathrm{~h}$, the cells were collected by centrifugation and were incubated in $500 \mu 1$ binding buffer supplemented with $5 \mu \mathrm{l}$ FITC-Annexin $\mathrm{V}$ and $5 \mu \mathrm{l}$ propidium iodide (PI). Fluorescence of the stained cells was then analyzed using flow cytometry.

Cell cycle analysis. Cells were collected as previously described and fixed in cold $70 \%$ ethanol overnight at $4^{\circ} \mathrm{C}$. After staining with PI and treatment with $100 \mu \mathrm{l}$ RNase A, the cells were kept in the dark at room temperature for $30 \mathrm{~min}$. The signals were analyzed using flow cytometry (BD FACSCalibur; BD Biosciences).

Tumor growth assay. All animal research was conducted using a protocol approved by the Animal Care and Use Committee of Nanjing Medical University (Nanjing, China). The animals were maintained at $25^{\circ} \mathrm{C}$ on a 12 -h light/dark cycle, and housed in a controlled environment and received food and water ad libitum. The animals were acclimated in rooms for 7 days before the initiation of the experiment.

A total of $1 \times 10^{6}$ cells (EC109 cells with stable expression of miR-125b, control cells or NC) were injected the left forelimb in nude mice. The nude mice $(25 \pm 5 \mathrm{~g})$ purchased from the Laboratory Animal Centre of Nanjing Medical University and 
A

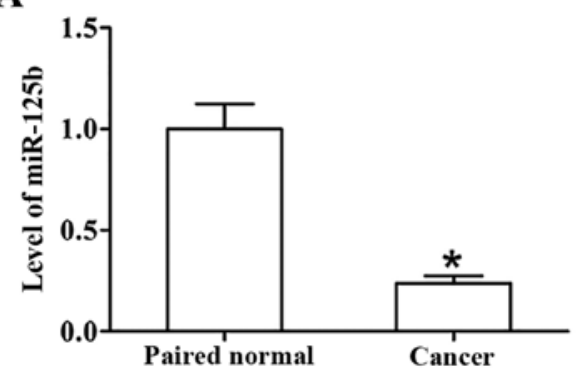

B

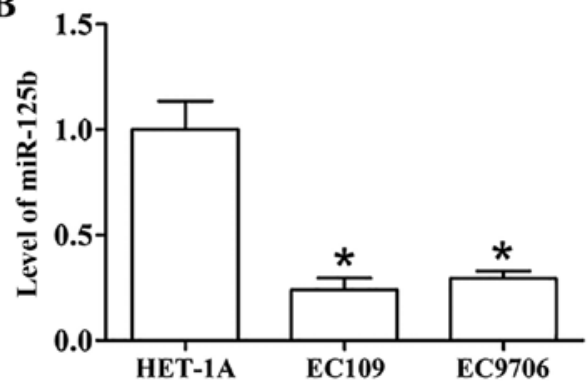

Figure 1. The expression of miR-125b in ESCC tissues and cells. (A) The expression of miR-125b in ESCC and paired normal tissues was examined by qRT-PCR. (B) The expression of miR-125b in ESCC cell lines and a human esophageal epithelial cell line was examined by qRT-PCR. *P<0.05 vs. the control. ESCC, esophageal squamous cell carcinoma.

used in the experiment were both male and female, at a ratio of 1:1. Six mice were assigned per group. Tumor volumes were assessed every 7 days. The mean tumor volume was calculated using the formula: [0.5 x (length $\mathrm{x}$ width $\left.\left.{ }^{2}\right)\right]$. After 28 days, the mice were sacrificed, and then tumors were dissected.

Mice were sacrificed using cervical dislocation euthanasia, when the following humane endpoints were present: i) tumor volumes reached $\geq 10 \%$ of the body weight of the mouse; ii) tumor length $\geq 20 \mathrm{~mm}$; iii) continuous body weight loss; iv) a rapid growth of the tumor to ulceration, causing infection or necrosis; and v) tumors of any size interfering with eating, drinking, urinating, defecating, or walking. No mice exhibited any signs of illness due tumor formation. No animals presented multiple tumors. Animal health observations were performed twice daily at 10 and $3 \mathrm{pm}$ by our husbandry staff.

Histological and immunohistochemical analyses. Tumor tissues were dissected and stained with hematoxylin and eosin (H\&E). For immunohistochemistry, tumor tissues were stained with Ki-67. The tissue apoptosis rate was assessed using the TdT-mediated dUTP nick and labeling (TUNEL) detection kit (Biotool, Houston, TX, USA), according to the manufacturer's instructions. Images were obtained using an Olympus microscope (Olympus, Tokyo, Japan).

Luciferase reporter assay. The mutant or wild-type 3'-UTR of BMF was amplified and cloned into the vector psiCHECK-2 to construct luciferase reporter plasmids. Cells ( $1 \times 10^{4} /$ well) were co-transfected with vectors and miR-125b mimics in each well. They were incubated in RPMI-1640 medium supplemented with $10 \%$ FBS. The luciferase activity was detected using the Dual-Luciferase Reporter Assay System (Promega Corp., Madison, WI, USA) after incubation for $48 \mathrm{~h}$. All the assays were performed in triplicate.

Western blotting. To assess the protein expression in tumor tissues and cells, total proteins were extracted using RIPA lysis buffer. The supernatant was collected by centrifugation at $13,282 \times \mathrm{g}$ for $15 \mathrm{~min}$. Next, the extracted proteins were incubated with the loading buffer at $100^{\circ} \mathrm{C}$ for $5 \mathrm{~min}$. Protein concentration was assessed using the Pierce BCA Protein Assay kit (Thermo Fisher Scientific, Inc.). Proteins were subjected to separation on $10 \%$ SDS-PAGE gels and transferred onto polyvinylidene fluoride (PVDF) membranes
(Milipore, Billerica, MA, USA), which were blocked using $5 \%(\mathrm{w} / \mathrm{v})$ non-fat dry milk. The membranes were probed using the following antibodies: rabbit anti-Bax antibody (1:2,000; cat. no. 2744), rabbit anti-Bcl-2 antibody (1:2,000; cat. no. 4223; both from Cell Signaling Technology, Inc., Danvers, MA, USA), rabbit anti-caspase-3 antibody (1:2,000; cat. no. ab13847; Abcam, Cambridge, UK) and rabbit anti-p27 antibody (1:2,000; cat. no. 3688; Cell Signaling Technology). The membranes were visualized using an ECL kit (Beyotime Institute of Biotechnology, Beijing, China). The amount of protein was normalized with respect to GAPDH and analyzed using the Image J software (NIH, Bethesda, MD, USA). All experiments were performed independently in triplicate.

Statistical analysis. GraphPad Prism 6 software was used to carry out all statistical analyses (GraphPad Software, Inc., La Jolla, CA, USA). When only two groups were compared, a Student's t-test was conducted. In addition, one-way ANOVA using Dunnett's multiple comparison test was applied to compare differences between multiple groups. A P-value of $<0.05$ was considered to indicate a statistically significant difference. In addition, the relationship between the expression of miR-125b and BMF was analyzed using Pearson's correlation analysis. All quantitative data are expressed as the mean $\pm \mathrm{SD}$.

\section{Results}

miR-125b is downregulated in ESCC tissues and cells. The level of miR-125b in clinical ESCC and normal tissues was assessed using real-time PCR. The results indicated that miR125 b expression was downregulated in the ESCC tumor tissues compared with paired normal tissues (Fig. 1A). Furthermore, compared to HET-1A, a normal esophageal epithelial cell line, the expression of miR-125b was lower in ESCC cell lines (EC109 and EC9706) (Fig. 1B). In addition, a decrease in the level of miR-125b was markedly associated with lymphatic metastasis in patients; Table I reveals the detailed clinical characteristics of patients. These results revealed that miR125 b may play a crucial role in ESCC carcinogenesis.

miR-125b suppresses proliferation in ESCC cells. To explore the roles of miR-125b in ESCC, human EC109 and EC9706 cells were transfected with miR-125b mimics or inhibitors. Compared with the control, transfection with miR-125b 
A

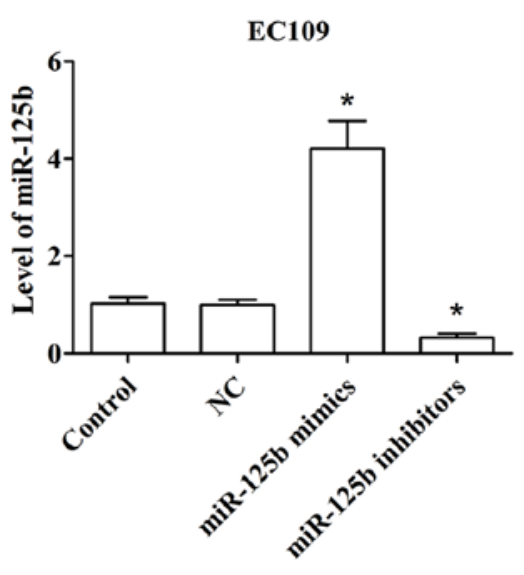

B

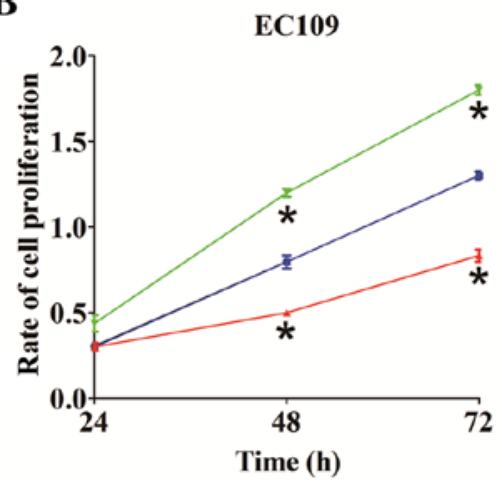

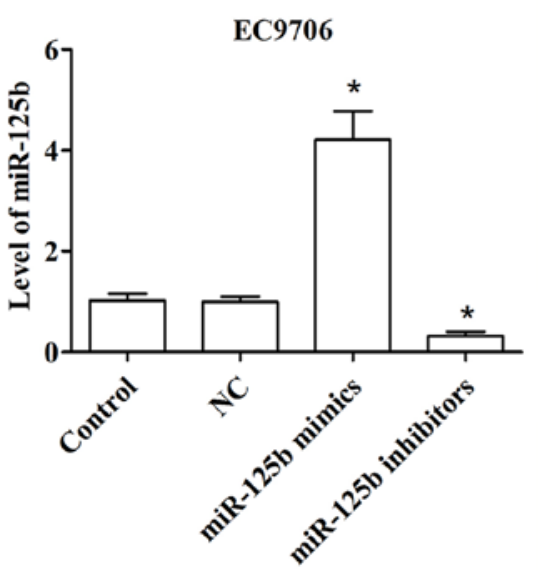

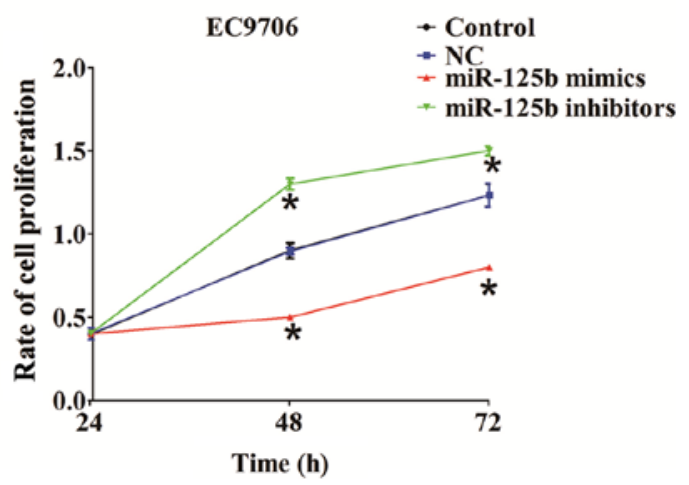

Figure 2. miR-125b inhibits ESCC cell proliferation. (A) EC109 and EC9706 cells transfected with miR-125b mimics or miR-125b inhibitors significantly increased or decreased, respectively, the expression of miR-125b compared with the control. (B) EC109 and EC9706 cells transfected with miR-125b mimics or miR-125b inhibitors both exhibited a significant decrease or increase, respectively, in the proliferation rate compared with the control. "P<0.05 vs. the control. ESCC, esophageal squamous cell carcinoma.
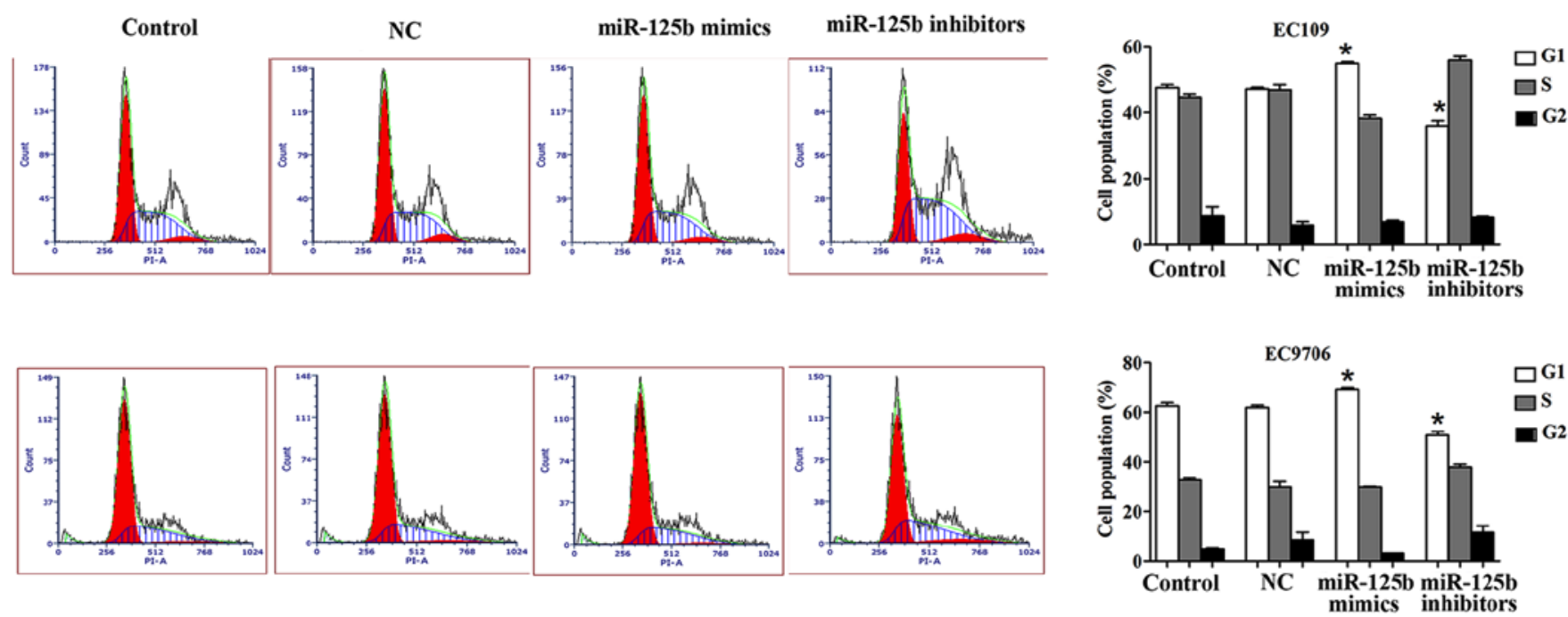

Figure 3. miR-125b inhibits the ESCC cell cycle. EC109 and EC9706 cells transfected with miR-125b mimics significantly increased the G1 phase of the cell cycle compared with the control. ${ }^{*} \mathrm{P}<0.05$ vs. the control. ESCC, esophageal squamous cell carcinoma.

mimics significantly upregulated miR-125b expression, while transfection with miR-125b inhibitors significantly downregulated miR-125b expression (Fig. 2A).

A CCK-8 assay was performed to assess the proliferation rate of EC109 and EC9706 cells transfected with miR-125b mimics or miR-125b inhibitors for 24,48 and $72 \mathrm{~h}$. Compared with the control, overexpressed miR-125b decreased cell proliferation in EC109 and EC9706 cells, whereas the growth of EC109 and EC9706 cells significantly increased posttransfection with miR-125b inhibitors (Fig. 2B). 
A
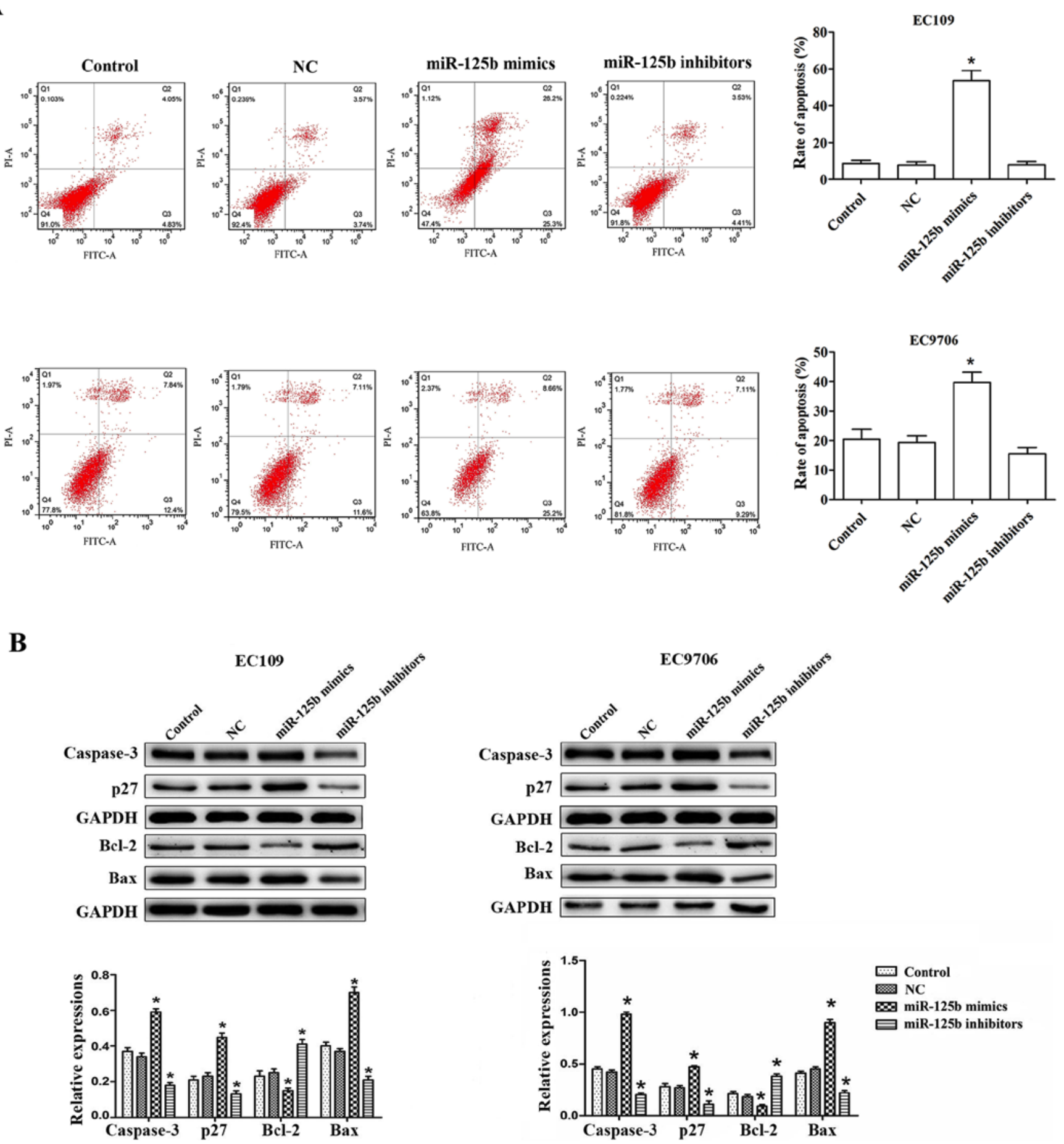

Figure 4. miR-125b induces ESCC cell apoptosis. (A) EC109 and EC9706 cells transfected with miR-125b mimics significantly increased cell apoptosis compared with the control. (B) The expression of caspase-3, Bcl-2, Bax and p27 were analyzed in ESCC cell lines. "P $<0.05$ vs. the control. ESCC, esophageal squamous cell carcinoma.

miR-125b induces cell cycle arrest in the G1 phase in ESCC. Flow cytometric analysis was performed to detect the effect of miR-125b on the cell cycle in EC109 and EC9706 cells. The results revealed that upregulation of miR-125b significantly increased the G1 phase of the cell cycle, while this phase was significantly decreased when the EC109 and EC9706 cells were transfected with miR-125b inhibitors compared with the control. Collectively, it was observed that miR-125b could arrest the cell cycle in the G1 phase in ESCC (Fig. 3).
miR-125b promotes apoptosis in ESCC cells. To determine whether the decrease in cell proliferation post-transfection with miR-125b mimic transfection was the result of apoptosis in EC109 and EC9706 cells, flow cytometric analysis was conducted to detect the apoptotic cells. For EC109 cells, the the proportion of apoptotic cells $(\mathrm{Q} 2+\mathrm{Q} 3)$ in the control group was $8.88 \pm 2.95 \%$, while the proportion of apoptotic cells (Q2+Q3) was $51.50 \pm 9.47 \%$ in the miR-125b mimic group. This revealed that the number of apoptotic cells markedly increased 
A

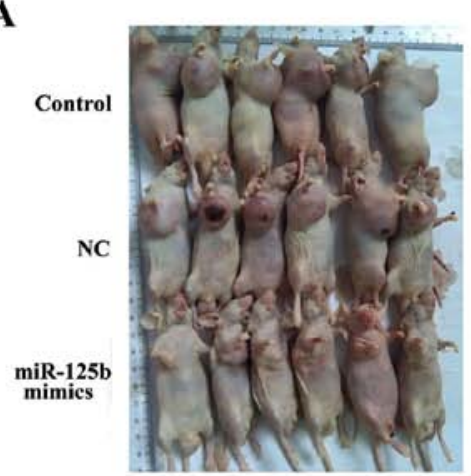

B

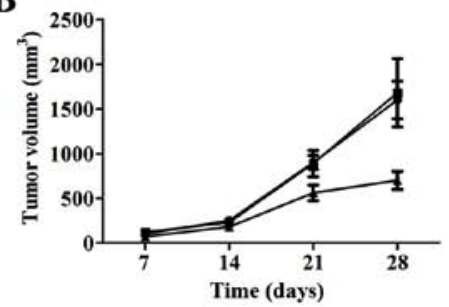

D
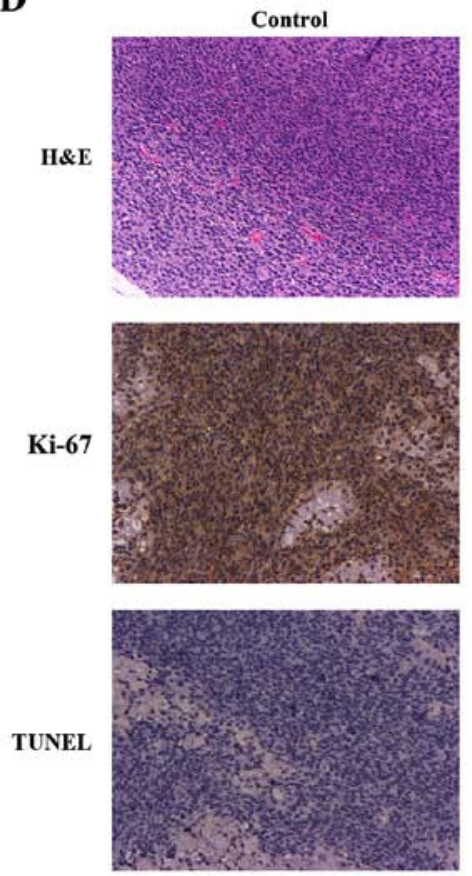

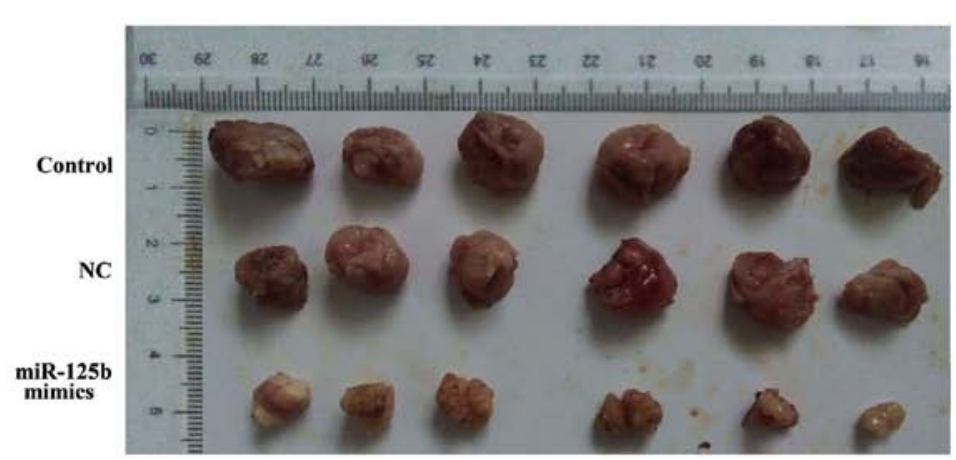

C

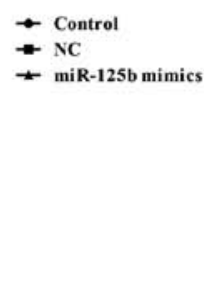

NC


miR-125b mimics
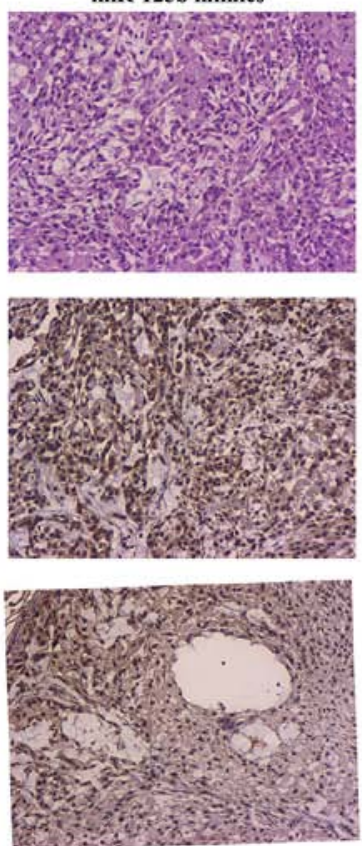

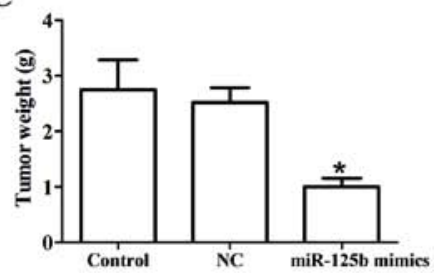

Figure 5. miR-125b suppresses EC109 cell growth in vivo. (A) EC109 cells (1x106/mouse) transfected with miR-125b mimics or NC were subcutaneously injected into the left forelimb of nude mice. (B) The tumor volumes were measured on the indicated days. (C) Excised tumor weight from the three separate groups was recorded. (D) H\&E staining, TUNEL and immunohistochemistry of Ki-67 from the tumor sections were assessed. ${ }^{*} \mathrm{P}<0.05$ vs. the control. $\mathrm{NC}$, negative control. H\&E, hematoxylin and eosin.

after the EC109 cells were transfected with miR-125b mimics. Similarly, the EC9706 cells after transfection with miR-125b mimics exhibited increased cell apoptosis compared with the control, while inhibition of miR-125b significantly decreased cell apoptosis (Fig. 4A).

Furthermore, the expression of apoptosis-related proteins, including Bax, Bcl-2, caspase-3 and p27, was analyzed. Of these proteins, 3 of the pro-apoptotic proteins, namely Bax, caspase-3 and p27, were observed to be upregulated in the EC109 and EC9706 cells transfected with miR-125b mimics in comparison with the cells of the control group. However, the anti-apoptotic protein Bcl-2 was significantly downregulated in cells transfected with miR-125b mimics. Additionally, the opposite results were obtained when the cells were transfected with miR-125b inhibitors. Thus, the levels of pro-apoptotic proteins were downregulated, while that of an anti-apoptotic protein was upregulated (Fig. 4B).

miR-125b inhibits tumor growth in vivo. We verified the role of miR-125b in ESCC using miR-125b mimics and miR-125b inhibitors, in vitro. The results revealed that overexpression of miR-125b inhibited cell proliferation and induced cell 
A

miR-125b 3' AGUGUUCAAUCCCA GAGUCCCU

BMF 5' CCACCUCCAGCAGACCUCAGGGA

B

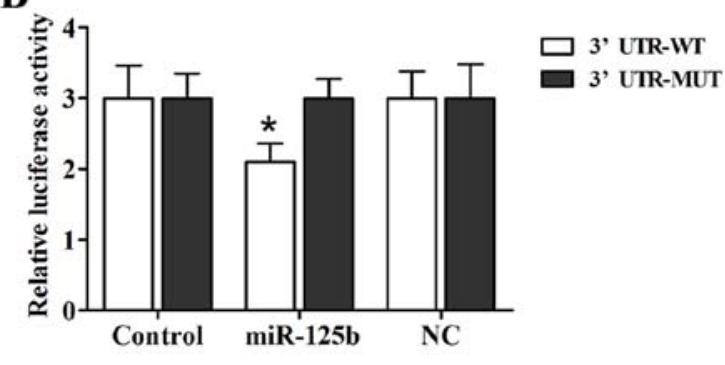

C

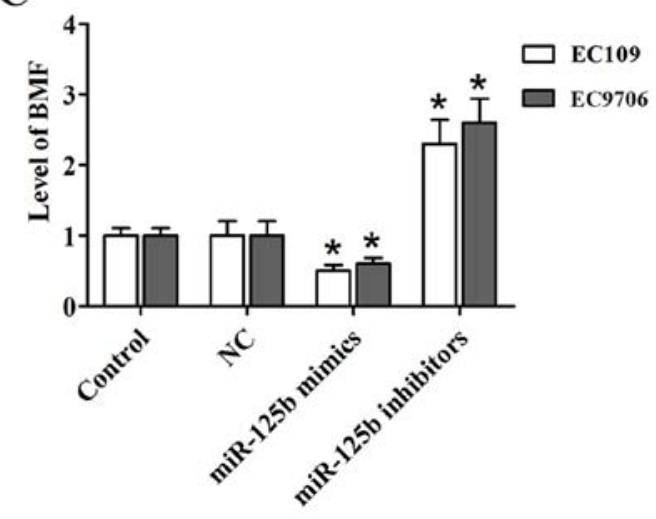

D

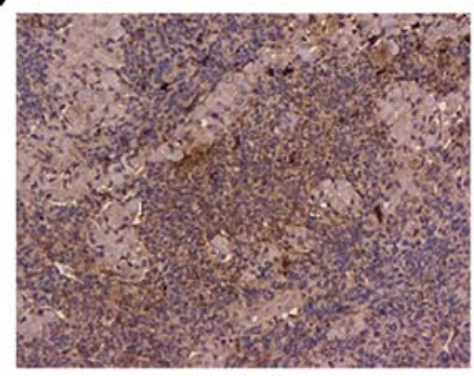

Control

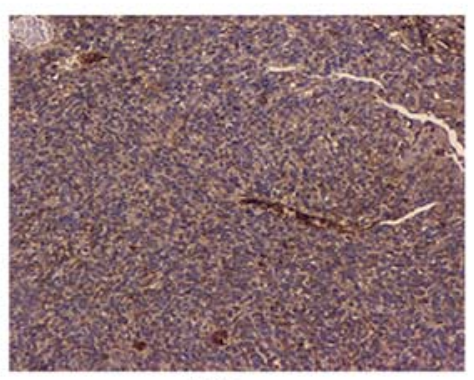

NC

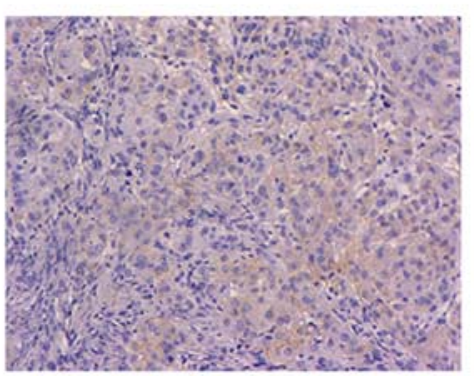

miR-125b

Figure 6. BMF is a direct target of miR-125b in ESCC cancer cells. (A) The prediction of the binding between miR-125b and BMF as determined using TargetScan. (B) A dual-luciferase reporter assay was performed to verify the binding of miR-125b with BMF. (C) qRT-PCR assay was performed to detect the mRNA level of BMF in EC109 and EC9706 cells treated with miR-125b mimics and miR-125b inhibitors. (D) The expression of BMF was assessed in the tumor sections. " $\mathrm{P}<0.05$ vs. the control. BMF, BCL-2-modifying factor; ESCC, esophageal squamous cell carcinoma.

apoptosis. Conversely, downregulation of miR-125b promoted cell proliferation and suppressed cell apoptosis. Thus, we next studied the inhibitory role of miR-125b in ESCC in vivo.

To investigate the potential effect of miR-125b on tumor growth in vivo, EC109 cells were transfected with miR-125b mimics or NC and injected into nude mice. In this experiment, one mouse reached a humane endpoint, and the percentage of the total number of animals used in the study was $4.17 \%$. After the end of the experiment, the maximum tumor burden in mice was $9.84 \%$ (the tumor weight was $3.12 \mathrm{~g}$ and the animal body weight was $31.7 \mathrm{~g}$ ), the longest diameter exhibited by a single subcutaneous tumor was $1.90 \mathrm{~cm}$, and none of the animals presented $>1$ tumor.

Compared with the control, miR-125b mimics markedly inhibited tumor growth. Furthermore, compared to the control group, the tumor weight of the miR-125b mimic group was remarkably lesser than the NC group. Additionally, compared to the control group, a lower expression of Ki-67 and a higher percentage of apoptotic cells were observed in the miR-125b mimic group using immunohistochemical staining (Fig. 5).

$B M F$ is a direct target gene of $m i R-125 b$. TargetScan was employed to search for the potential target genes of miR-125b.
It identified BMF as a candidate target gene (Fig. 6A). The Dual-Luciferase reporter assay was performed to confirm this hypothesis (Fig. 6B). Furthermore, we examined whether the expression of BMF was affected by miR-125b in ESCC cells. In our study, we observed that the level of BMF was significantly reduced in the EC109 and EC9706 cells transfected with miR-125b mimics (Fig. 6C). In the in vivo tumor growth experiment, immunohistochemical analysis of the tumor sections revealed decreased expression of BMF in the miR-125b mimic group (Fig. 6D).

Silencing of BMF suppresses cell proliferation and induces apoptosis in ESCC. To clarify whether BMF was involved in regulating ESCC cell proliferation and apoptosis, we knocked down its expression by transfecting the EC109 and EC9706 cells with si-BMF. qRT-PCR and western blotting were performed to assess the transfection efficiency. Compared to the control, the expression of BMF was markedly downregulated in the EC109 and EC9706 cells transfected with si-BMF (Fig. 7A and B).

Cell proliferation was evaluated using the CCK-8 assay. EC109 and EC9706 cells transfected with si-BMF exhibited 

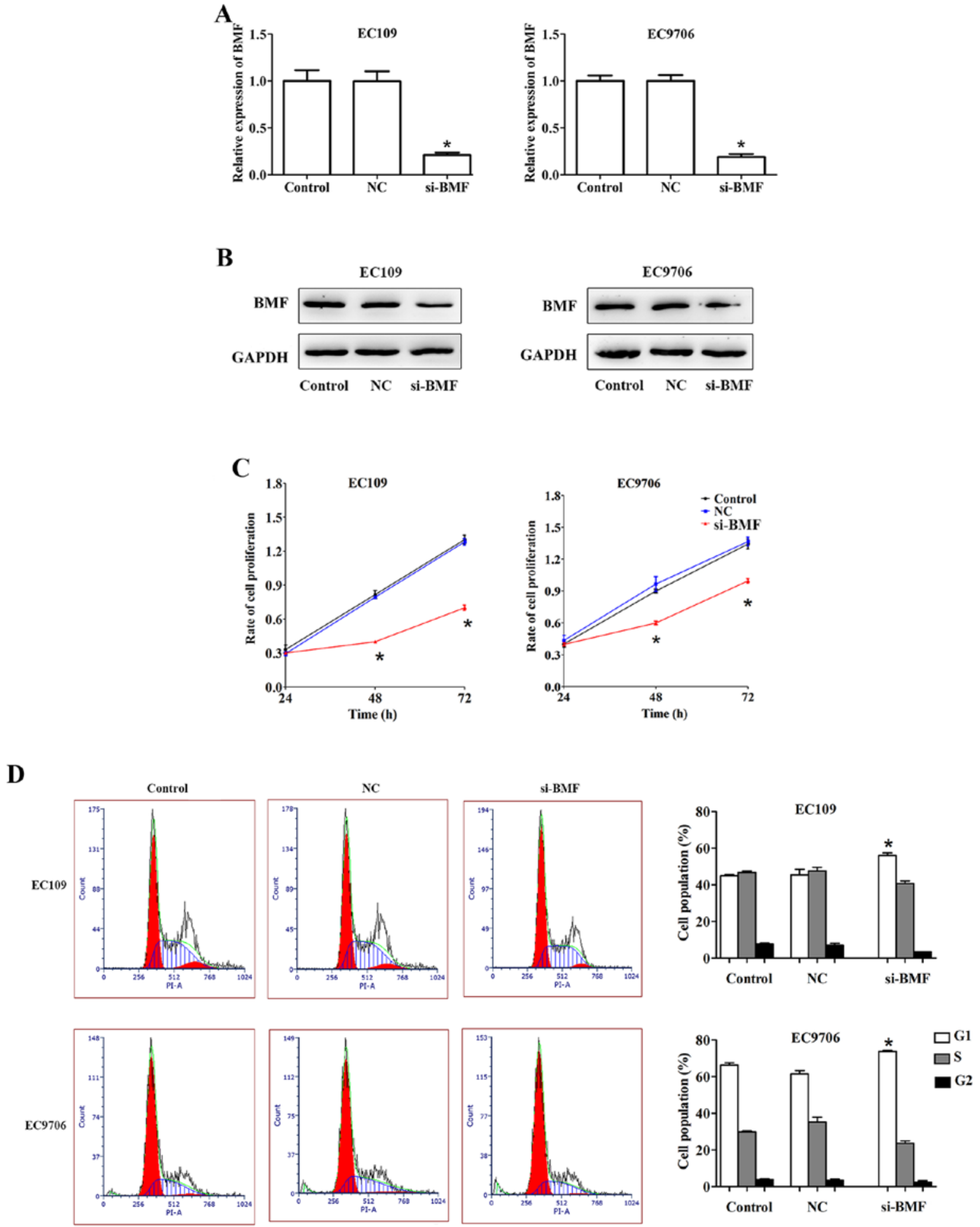

Figure 7. BMF inhibits ESCC cell proliferation. (A) A qRT-PCR assay was conducted to assess the mRNA expression of BMF. (B) Western blot analysis was performed to assess the protein expression of BMF. (C) A CCK-8 assay was used to reveal the proliferation rate in ESCC cells with si-BMF transfection. (D) The cell cycle was examined in ESCC cell lines. ${ }^{*} \mathrm{P}<0.05$ vs. the control. BMF, BCL-2-modifying factor; ESCC, esophageal squamous cell carcinoma.

slower growth than the control cells (Fig. 7C). Moreover, compared to the control, the si-BMF group exhibited an increase in the G1 phase of the cell cycle in EC109. Similar results were obtained for the EC9706 cells (Fig. 7D). BMF silencing notably promoted cell apoptosis in EC109 and EC9706 cells. For EC109 cells, the proportion of apoptotic cells $(\mathrm{Q} 2+\mathrm{Q} 3)$ was $8.09 \pm 1.96 \%$ in the control group, while the proportion of apoptotic cells $(\mathrm{Q} 2+\mathrm{Q} 3)$ was $30.30 \pm 5.61 \%$ in the si-BMF group thus, revealing a significant increase in apoptotic cells. Similar results were obtained for the EC9706 cells (Fig. 8A). Western blot analysis indicated that BMF silencing markedly increased the expression of Bax, caspase-3 and p27, and decreased that of Bcl-2 in ESCC cells (Fig. 8B). Collectively, these results revealed that BMF participated in the miR-125b-mediated regulation of ESCC cell proliferation, the cell cycle and apoptosis. 
A
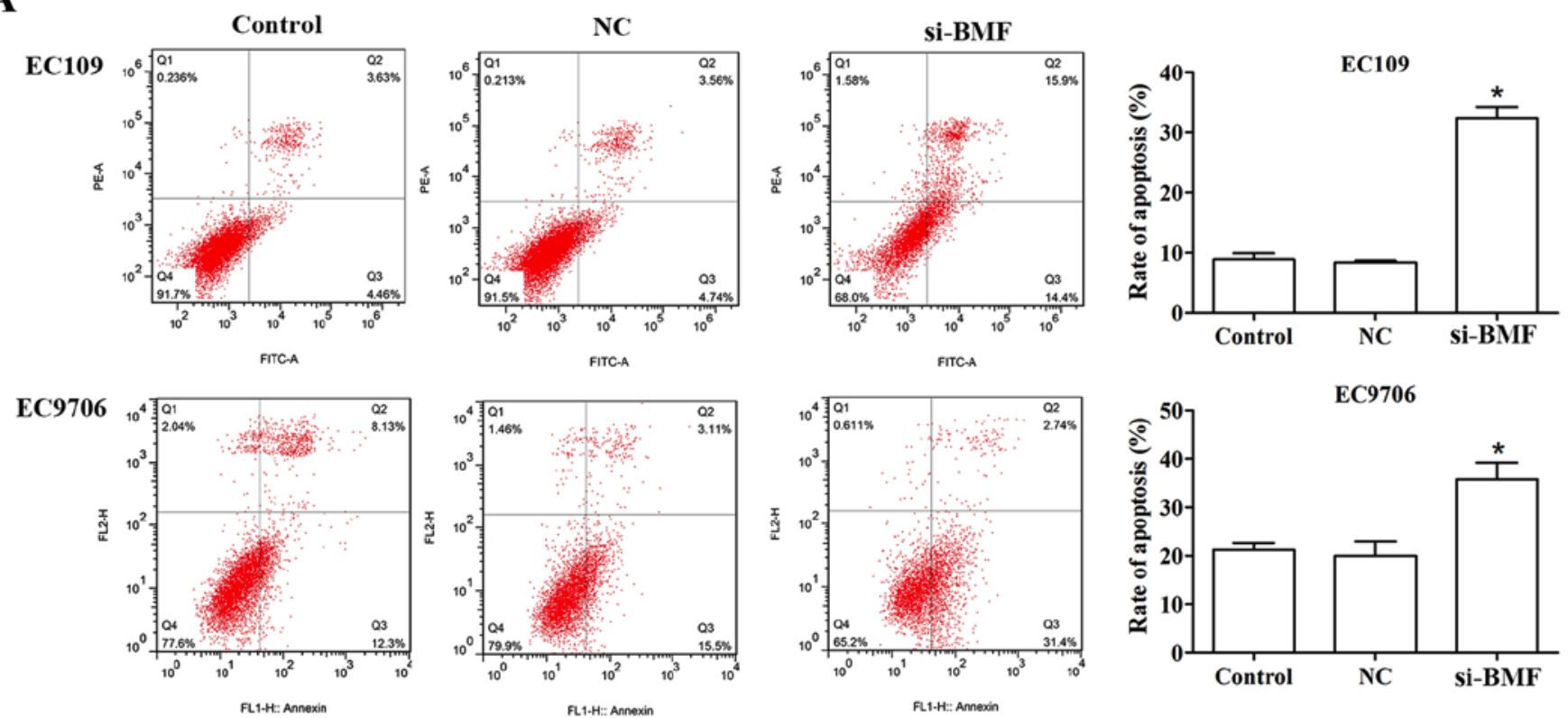

\section{B}
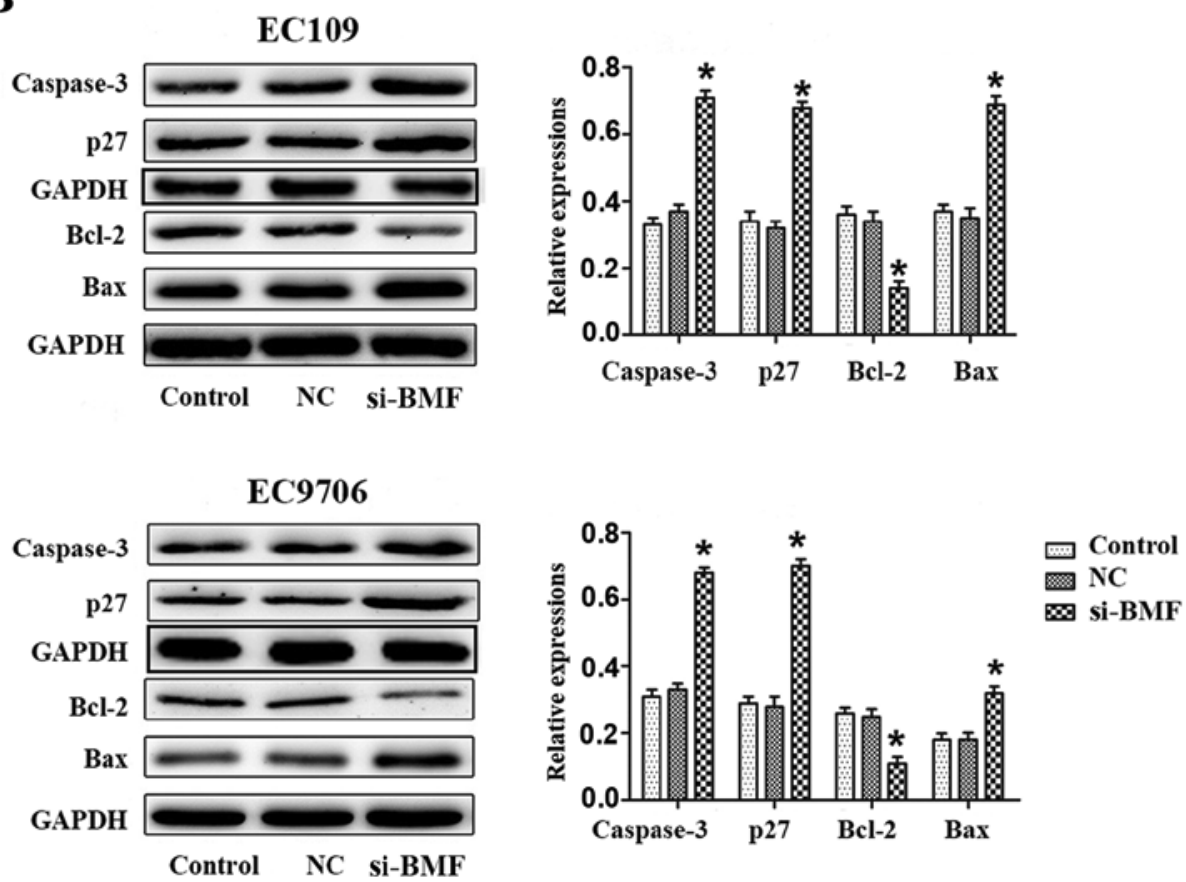

Figure 8. BMF induces ESCC cell apoptosis. (A) Cell apoptosis was assayed in ESCC cell lines. (B) The protein level was assayed by western blotting in ESCC cell lines ${ }^{*} \mathrm{P}<0.05$ vs. the control. BMF, BCL-2-modifying factor; ESCC, esophageal squamous cell carcinoma.

The expression level of miR-125b is negatively correlated with that of BMF in ESCC. The relationship between BMF and miR-125b was further confirmed. We assessed the expression of BMF in tissues of ESCC patients and ESCC cell lines. The results indicated that BMF was increasingly upregulated in tumor tissues than in the adjacent non-cancerous tissues (Fig. 9A and C). We further observed that the levels of BMF in EC109 and EC9706 were in accordance with the tissues (Fig. 9B and D). In addition, we also explored the relationship between BMF and miR-125b. The result revealed a negative correlation between miR-125b and BMF levels (Fig. 9E).

\section{Discussion}

Accumulating evidence has revealed that miRNAs are closely associated with the initiation and progression of ESCC by activating or suppressing multiple malignant processes $(22,23)$. However, the mechanisms underlying ESCC pathogenesis have not been fully elucidated. In the present study, miR-125b expression was observed to be considerably low in ESCC tissues and cells. Furthermore, low miR-125b expression in tumors was significantly correlated with lymphatic metastasis in patients, thus revealing that miR-125b may be a useful 


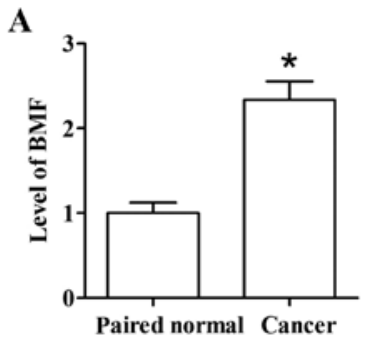

C



D

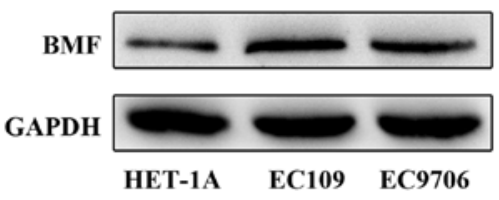

$\mathbf{E}$

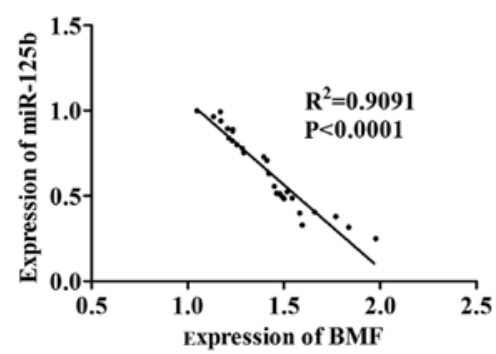

B
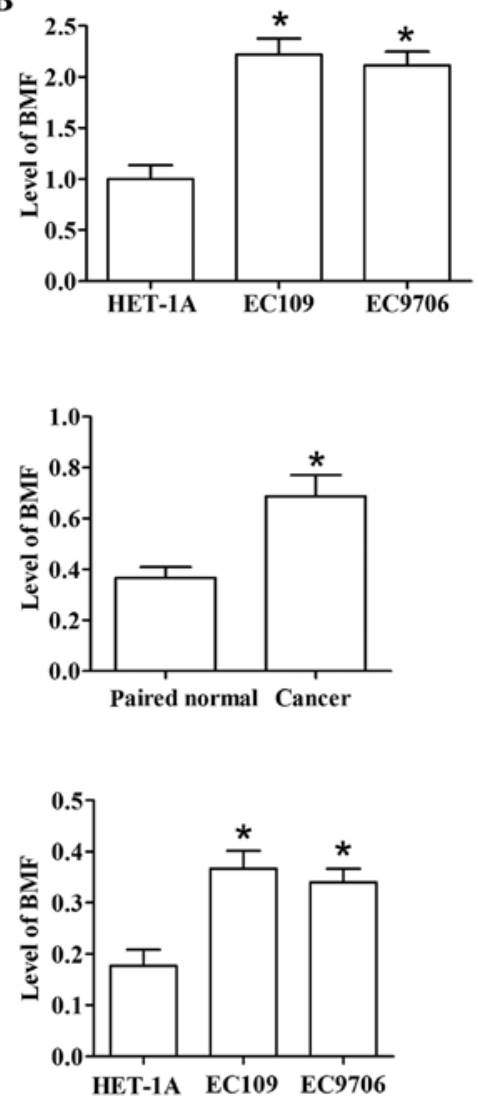

Figure 9. Relationship between miR-125b and BMF in ESCC. (A) The mRNA expression of BMF in ESCC tissues compared to normal tissues. (B) The mRNA expression of BMF in ESCC cell lines (EC109 and EC9706 cells) compared to an esophageal epithelial cell line (HET-1A). (C) The protein expression of BMF in ESCC tissues compared to normal tissues. (D) The protein expression of BMF in ESCC cells (EC109 and EC9706 cells) compared to an esophageal epithelial cell line (HET-1A). (E) Data analysis of relationship between the expression of miR-125b and BMF in ESCC tissues. "P<0.05 vs. the control. BMF, BCL-2-modifying factor; ESCC, esophageal squamous cell carcinoma.

prognostic biomarker. It was also observed that the level of miR-125b was closely associated with ESCC tumorigenesis. We demonstrated that the overexpression of miR-125b suppressed cell proliferation, and induced cell cycle arrest, and promoted apoptosis in ESCC lines EC109 and EC9706, which have been widely used in research of esophageal squamous cell cancer $(24,25)$. Additionally, overexpression of miR-125b markedly inhibited tumor cell growth in vivo. Furthermore, we observed that miR-125b directly targeted BMF in ESCC cells. Notably, by decreasing the level of BMF similar outcomes as with the overexpression of miR-125b were obtained.

Aberrant expression of miRNAs has been revealed in several types of cancer $(26,27)$. Therefore, a better understanding of the mechanisms underlying miRNA-mediated regulation networks is vital for developing diagnostic and therapeutic strategies $(28,29)$. Herein, miR-125b expression was observed to be markedly decreased in ESCC. Previous studies have reported the functions of miR-125b in other types of cancers, including gallbladder and colorectal cancer, and melanoma (30-32). miR-125b had the ability to regulate cell proliferation, differentiation and invasion $(33,34)$, and potentially act as an tumor suppressor in some cancer types, suppressing tumorigenesis and development $(35,36)$. We found that miR-125b was significantly reduced in thyroid cancer and oral squamous cell carcinoma $(37,38)$, indicating that it may act as a tumor suppressor. miR-125b was found to be markedly upregulated in the prostate, and it inhibited cancer cell proliferation by suppressing the expression of BAK1. However, the molecular mechanism of miR-125b in ESCC pathogenesis remains largely unknown.

In the present study, BMF was validated as a direct target of miR-125b. It was observed to be frequently overexpressed 
in a variety of tumors (39). As an oncogene, BMF participates in the pathogenesis of several tumors. In human E-cadherinnegative breast cancer, BMF promoted tumor growth and metastasis, and was activated by the transcription factor FOXO3 (40). In colon cancer, BMF promoted cell proliferation and inhibited apoptosis, and was activated by the transcription factor Eomes (39). Consequently, BMF is a member of the pro-apoptotic Bcl-2 family, which is mainly associated with cell proliferation and apoptosis. In the present study, the results revealed that silencing BMF could markedly inhibit cell growth and promote cell apoptosis in EC109 and EC9706 cells.

In summary, the results indicated that knockdown of miR-125b expression may prevent ESCC tumor initiation and progression by controlling BMF levels. This may be used as a potential therapeutic strategy to inhibit ESCC progression.

\section{Acknowledgements}

Not applicable.

\section{Funding}

No funding was received.

\section{Availability of data and materials}

The datasets used during the present study are available from the corresponding author upon reasonable request.

\section{Authors' contributions}

YXF and XHB conceived and designed the study. PDQ, ZZC, JW and QZ performed the experiments. YXF and QZ wrote the manuscript. YHL and PWY reviewed and edited the manuscript. All authors read and approved the manuscript and agree to be accountable for all aspects of the research in ensuring that the accuracy or integrity of any part of the report work are appropriately investigated and resolved.

\section{Ethics approval and consent to participate}

All experimental protocols were approved by the Ethics Committee of Jiangsu Cancer Hospital (Nanjing, Jiangsu, China).

\section{Consent for publication}

Not applicable.

\section{Competing interests}

The authors declare that they have no competing interests.

\section{References}

1. Enzinger PC and Mayer RJ: Esophageal cancer. N Engl J Med 349: 2241-2252, 2003

2. Kollarova H, Machova L, Horakova D, Janoutova G and Janout V: Epidemiology of esophageal cancer - an overview article. Biomed Pap Med Fac Univ Palacky Olomouc Czech Repub 151: 17-20, 2007.
3. Bohanes P, Yang D, Chhibar RS, Labonte MJ, Winder T, Ning Y, Gerger A, Benhaim L, Paez D, Wakatsuki T, et al: Influence of sex on the survival of patients with esophageal cancer. J Clin Oncol 30: 2265-2272, 2012

4. Kamangar F, Dores GM and Anderson WF: Patterns of cancer incidence, mortality, and prevalence across five continents: Defining priorities to reduce cancer disparities in different geographic regions of the world. J Clin Oncol 24: 2137-2150, 2006 .

5. Pennathur A, Gibson MK, Jobe BA and Luketich JD: Oesophageal carcinoma. Lancet 381: 400-412, 2013.

6. Zhao P, Dai M, Chen W and Li N: Cancer trends in China. Jpn J Clin Oncol 40: 281-285, 2010.

7. Liu J, Xie X, Zhou C, Peng S, Rao D and Fu J: Which factors are associated with actual 5-year survival of oesophageal squamous cell carcinoma? Eur J Cardiothorac Surg 41: e7-e11, 2012.

8. Garzon R, Calin GA and Croce CM: MicroRNAs in Cancer. Annu Rev Med 60: 167-179, 2009.

9. Nana-Sinkam SP and Croce CM: Clinical applications for microRNAs in cancer. Clin Pharmacol Ther 93: 98-104, 2013.

10. Lu J, Getz G, Miska EA, Alvarez-Saavedra E, Lamb J, Peck D, Sweet-Cordero A, Ebert BL, Mak RH, Ferrando AA, et al: MicroRNA expression profiles classify human cancers. Nature 435: 834-838, 2005.

11. Yi Y, Lu X, Chen J, Jiao C, Zhong J, Song Z, Yu X and Lin B: Downregulated miR-486-5p acts as a tumor suppressor in esophageal squamous cell carcinoma. Exp Ther Med 12: 3411-3416, 2016

12. Ferracin M, Veronese A and Negrini M: Micromarkers: miRNAs in cancer diagnosis and prognosis. Expert Rev Mol Diagn 10: 297-308, 2010.

13. Ren LH, Chen WX, Li S, He XY, Zhang ZM, Li M, Cao RS Hao B, Zhang HJ, Qiu HQ, et al: MicroRNA-183 promotes proliferation and invasion in oesophageal squamous cell carcinoma by targeting programmed cell death 4. Br J Cancer 111: 2003-2013, 2014.

14. Wang J, Tian X, Han R, Zhang X, Wang X, Shen H, Xue L, Liu Y, Yan X, Shen J, et al: Downregulation of miR-486-5p contributes to tumor progression and metastasis by targeting protumorigenic ARHGAP5 in lung cancer. Oncogene 33: 1181-1189, 2104, 2014.

15. Zhang G, Liu Z, Cui G, Wang X and Yang Z: MicroRNA-486-5p targeting PIM-1 suppresses cell proliferation in breast cancer cells. Tumour Biol 35: 11137-11145, 2104.

16. Huang XP, Hou J, Shen XY, Huang CY, Zhang XH, Xie YA and Luo XL: MicroRNA-486-5p, which is downregulated in hepatocellular carcinoma, suppresses tumor growth by targeting PIK3R1. FEBS J 282: 579-594, 2015.

17. Ferretti E, De Smaele E, Miele E, Laneve P, Po A, Pelloni M, Paganelli A, Di Marcotullio L, Caffarelli E, Screpanti I, et al: Concerted microRNA control of Hedgehog signalling in cerebellar neuronal progenitor and tumour cells. EMBO J 27: 2616-2627, 2008

18. Shi XB, Xue L, Ma AH, Tepper CG, Kung HJ and White RW: miR-125b promotes growth of prostate cancer xenograft tumor through targeting pro-apoptotic genes. Prostate 71: 538-549, 2011.

19. Fujino Y, Takeishi S, Nishida K, Okamoto K, Muguruma N, Kimura T, Kitamura S, Miyamoto H, Fujimoto A, Higashijima J, et al: Downregulation of $\mathrm{miR}-100 / \mathrm{miR}-125 \mathrm{~b}$ is associated with lymph node metastasis in early colorectal cancer with submucosal invasion. Cancer Sci 108: 390-397, 2017.

20. Sun Y, Liu X, Zhang Q, Mao X, Feng L, Su P, Chen H, Guo Y and Jin F: Oncogenic potential of TSTA3 in breast cancer and its regulation by the tumor suppressors miR-125a-5p and miR-125b. Tumour Biol 37: 4963-4972, 2016.

21. Gu J, Wang Y and Wu X: MicroRNA in the pathogenesis and prognosis of esophageal cancer. Curr Pharm Des 19: 1292-1300, 2013.

22. Sakai NS, Samia-Aly E, Barbera M and Fitzgerald RC: A review of the current understanding and clinical utility of miRNAs in esophageal cancer. Semin Cancer Biol 23: 512-521, 2013.

23. Mayne GC, Hussey DJ and Watson DI: MicroRNAs and esophageal cancer - implications for pathogenesis and therapy. Curr Pharm Des 19: 1211-1226, 2013.

24. Jin YY, Chen QJ, Xu K, Ren HT, Bao X, Ma YN, Wei Y and Ma HB: Involvement of microRNA-141-3p in 5-fluorouracil and oxaliplatin chemo-resistance in esophageal cancer cells via regulation of PTEN. Mol Cell Biochem 422: 161-170, 2016.

25. Han N, Zhao W, Zhang Z and Zheng P: MiR-328 suppresses the survival of esophageal cancer cells by targeting PLCE1. Biochem Biophys Res Commun 470: 175-180, 2016. 
26. Chen L, Wang Q, Wang GD, Wang HS, Huang Y, Liu XM and Cai XH: miR-16 inhibits cell proliferation by targeting IGF1R and the Raf1-MEK1/2-ERK1/2 pathway in osteosarcoma. FEBS Lett 587: 1366-1372, 2013.

27. Yan K, Gao J, Yang T, Ma Q, Qiu X, Fan Q and Ma B: MicroRNA-34a inhibits the proliferation and metastasis of osteosarcoma cells both in vitro and in vivo. PLoS One 7: e33778, 2012.

28. Duan Z, Choy E, Harmon D, Liu X, Susa M, Mankin H and Hornicek F: MicroRNA-199a-3p is downregulated in human osteosarcoma and regulates cell proliferation and migration. Mol Cancer Ther 10: 1337-1345, 2011.

29. Jianwei Z, Fan L, Xiancheng L, Enzhong B, Shuai L and Can L: MicroRNA 181a improves proliferation and invasion, suppresses apoptosis of osteosarcoma cell. Tumour Biol 34: 3331-3337, 2013

30. Yang D, Zhan M, Chen T, Chen W, Zhang Y, Xu S, Yan J, Huang Q and Wang J: miR-125b-5p enhances chemotherapy sensitivity to cisplatin by down-regulating $\mathrm{Bcl} 2$ in gallbladder cancer. Sci Rep 7: 43109, 2017.

31. Yu X, Shi W, Zhang Y, Wang X, Sun S, Song Z, Liu M, Zeng Q, Cui $\mathrm{S}$ and Qu X: CXCL12/CXCR4 axis induced miR-125b promotes invasion and confers 5-fluorouracil resistance through enhancing autophagy in colorectal cancer. Sci Rep 7: 42226, 2017.

32. Pei G, Lan Y, Chen D, Ji L and Hua ZC: FAK regulates E-cadherin expression via p-Src ${ }^{\mathrm{Y} 416} / \mathrm{p}-\mathrm{ERK}_{1 / 2} / \mathrm{p}-\mathrm{Stat} 3^{\mathrm{Y} 705}$ and PPAR $\gamma / \mathrm{miR}-125 \mathrm{~b} / \mathrm{Stat} 3$ signaling pathway in B16F10 melanoma cells. Oncotarget 8: 13898-13908, 2017.

33. Bousquet M, Quelen C, Rosati R, Mansat-De Mas V, La Starza R, Bastard C,Lippert E, Talmant P, Lafage-Pochitaloff M, Leroux D, et al: Myeloid cell differentiation arrest by miR-125b-1 in myelodysplastic syndrome and acute myeloid leukemia with the $t(2 ; 11)$ (p21;q23) translocation. J Exp Med 205: 2499-2506, 2008.

34. Mizuno Y, Yagi K, Tokuzawa Y, Kanesaki-Yatsuka Y, Suda T, Katagiri T, Fukuda T, Maruyama M, Okuda A, Amemiya T, et al: miR-125b inhibits osteoblastic differentiation by downregulation of cell proliferation. Biochem Biophys Res Commun 368: 267-272, 2008.
35. Scott GK, Goga A, Bhaumik D, Berger CE, Sullivan CS and Benz CC: Coordinate suppression of ERBB2 and ERBB3 by enforced expression of micro-RNA miR-125a or miR-125b. J Biol Chem 282: 1479-1486, 2007.

36. Visone R, Pallante P, Vecchione A, Cirombella R, Ferracin M, Ferraro A, Volinia S, Coluzzi S, Leone V, Borbone E, et al: Specific microRNAs are downregulated in human thyroid anaplastic carcinomas. Oncogene 26: 7590-7595, 2007.

37. Zhang Y, Yan LX, Wu QN, Du ZM, Chen J, Liao DZ, Huang MY, Hou JH, Wu QL, Zeng MS, et al: miR-125b is methylated and functions as a tumor suppressor by regulating the ETS1 protooncogene in human invasive breast cancer. Cancer Res 71: 3552-3562, 2011

38. Gefen N, Binder V, Zaliova M, Linka Y, Morrow M, Novosel A, Edry L, Hertzberg L, Shomron N, Williams O, et al: Hsa-mir-125b-2 is highly expressed in childhood ETV6/ RUNX1 (TEL/AML1) leukemias and confers survival advantage to growth inhibitory signals independent of p53. Leukemia 24: 89-96, 2010.

39. Wang R, Kang Y, Löhr CV, Fischer KA, Bradford CS, Johnson G, Dashwood WM, Williams DE, Ho E and Dashwood RH: Reciprocal regulation of BMF and BIRC5 (Survivin) linked to Eomes overexpression in colorectal cancer. Cancer Lett 381: 341-348, 2016.

40. Hornsveld M, Tenhagen M, van de Ven RA, Smits AM, van Triest MH, van Amersfoort M, Kloet DE, Dansen TB, Burgering BM and Derksen PW: Restraining FOXO3-dependent transcriptional BMF activation underpins tumour growth and metastasis of E-cadherin-negative breast cancer. Cell Death Differ 23: 1483-1492, 2016.

This work is licensed under a Creative Commons Attribution-NonCommercial-NoDerivatives 4.0 International (CC BY-NC-ND 4.0) License. 